\title{
Holographic Spinal Scan Projection through Microsoft HoloLens
}

\author{
Pei Wen Voon¹, Ke Wang1, Gabriel Yin Foo Lee², Lei Cui ${ }^{*}$ \\ ${ }^{1}$ School of Civil \& Mechanical Engineering, Curtin University, Perth, WA 6102, Australia. \\ 2 SJOG Subiaco Hospital, Curtin University, University of Western Australia, Perth 6008, Australia. \\ * Corresponding author. Email: lei.cui@curtin.edu.au \\ Manuscript submitted May 10, 2018; accepted July 23, 2018. \\ doi: 10.17706/ijcee.2018.10.4.274-283
}

\begin{abstract}
Augmented reality (AR) technology has incorporated refined sensory perception into complex neurosurgical procedures in recent years. Practitioners used head mounted displays to access neuro-navigation in surgical preparations in pursuit of maximum safety. This paper was aimed to converge a target reference from imagery to an actual object by using Microsoft HoloLens. Reference registration is achieved through object scanning that utilizes phase detection of a sensor module to assign distinctive digital reference points. The navigation system then correlates the digital data with formulated computed tomography (CT) scans to accurately overlay holographic projections. Aside from sectioning the spine model projection, the interactive capability of the system extends through motion gesture and voice control to further emphasize on the hands-free experience in neutralizing surgical tension. Testing showed promising visual accuracy with fast processing response. This work explored the possibility of conducting neuro-navigation without reliance of an additional image guide.
\end{abstract}

Key words: AR spinal registration, microsoft Hololens, vuforia, mobile object scanner, spinal scan projection.

\section{Introduction}

The impressive development of computer-aided navigational system for the past decade has greatly influenced the adaptation of surgical applications. Though incorporation of the system does not impose significant impact upon overall system efficiency, it refines the aesthetic and functional aspects of surgical procedures [1]. Surgeons, granted with visual access of 3D anatomical structures, formulate from CT and MRI scans without concern of radiation exposure. Intrusive surgeries with high risk factors were especially reliant on the system to formulate and assist [2]. The growing need of posterior spinal procedures prompt a clinical study that indicates significant accuracy improvements with computer-assisted technique implementations [3].

In a 2006 study on adult tethered cord syndrome, the largest data series were collected through multimodality intraoperative neurophysiologic monitoring. Results were able to conclude useful adjunct to complex microsurgery through the SSEPs recordings in concert with continuous and evoked EMGs [4]. The constant development of navigational systems indicates the clinical potential for various neuro-navigational pursuits. Clinically acclaimed navigational systems such as MATRIX POLAR [5], Virtual Fluoroscopy [6] and $\mathrm{X}$-Scope [7] were commercialized. However, general commercialized navigational systems utilize monitors to render information, forcing surgeons to alternate line of vision between operating table and monitor 
screen. This complicates and prolongs the surgical procedures.

The rise of augmented reality in recent years has introduced head mounted display (HMD) as a probable medium for medical imaging diagnosis. Many researchers and professionals experimented with the offered target tracking application to explore the extent of device's clinical adaptability [8]. Most of the early built AR systems prioritised on educational simulations to cope with the rapid shift of neurosurgical practices. ImmersiveTouch, an AR simulation platform, uses multiple sensory modalities to simulate environmental cues expected from an actual surgical procedure. Available spinal modules includes pedicle screw placement, vertebroplasty, and lumbar puncture [9]. A 2014 publication indicates Google Glass as the first HMD involved in the surgical case adoption with integration of both VR and AR capabilities. The application also shows promise in field operatives by allowing interactive data transmission among surgeons at different locations [10].

Recent publications noted the establishment of HMD in AR neurosurgical treatments through the seamless fusion of cutting-edge optics and target sensors. It serves as a navigational portal that permits quantitative evaluation during pre-surgery phases [11] while eliminating visual diversion during surgical procedures [12], [13]. The HMD further demonstrates the capability of incorporating adaptive imaging technique for endoscopic image enhancement with color transform and grey level co-occurrence matrices to form a uniquely designed target marker [14]. In order to access additional functionalities, AR software adapted novel preoperative planning from various software development kit (SDK to facilitate relevant anatomy throughout the paranasal sinuses and adjacent structures [15]. Modelled projection could further enhance safety measures with each component segments, colors, and life-size visualizations tailored in accordance to the surgical requirements.

Microsoft HoloLens, the world's first fully untethered holographic computer, was deemed a qualified AR asset for clinical use through evaluation of comfort and hardware setup [16]. It redefined personal computing for autopsy, gross and microscopic examination by integrating refined sensory perception [17]. HoloLens permits a wide range of SDK installations, with popular options such as Vuforia, HolotoolKit, and MixedRealityToolKit-Unity. The Vuforia engine categorizes target genre into imagery, cuboid and physical object. Selection varies from every intended purposes. Imagery target is popularized by the minimalized localization error and fast response achievement of image-guided navigational system (IGNS) [18]. Collaborative features are implemented in consideration of data conversion fluidity evaluation [19]. Operative development platforms with distinctive command structures and interactive functionality are favored for their capability in maintaining robust battery life, sterility, and ideal form factor that emphasize intraoperative use [20].

The target accuracy is influenced by the following aspects: target placement, user location, lighting consistency, equipment involved and real-timed coordinate resolution [21]. Dependence of IGNS on coordinate frame registry renders the system vulnerable to risks of human and instrumental errors. For fiducials registration that attaches markers manually in simulation of physical-guide navigation, the practice has been found with less accuracy than frame-based methods [22]. Expansion of AR target variety later inspired the adoption of object target registration as a potential replacement for image-guided neuro-navigation system. The method differs from the traditional surface alignment approach by anchoring projection to the distinguishable feature of the intended target.

This paper describes the construction of target tracking system through instructive SDKs implementation in target identification and data manipulation to create a HoloLens application that features spinal structure identification capabilities. It presents the application development process from target registration to final HMD deployment in Section 2 while showcasing the deployment result in Section 3. Discussion in Section 4 highlights the spatial augmentation alignment and overall system latency. The paper 
ends by concluding findings in Section 5 .

\section{Methodology}

The system assembly revolves around Unity project development platform and various SDKs. Production of data target registration requires the assist of mobile scanner. Intended devices were either updated to version greater than 4.2 or equipped with 16-megapixel sensor and f/2.2 lens to qualify for target registration. Finalized application was assembled in Unity development platform then deployed through Visual studios. Caution upon respective system installation as system edition significantly impacts the compatibility of the implemented platforms and written scripts. Microsoft HoloLens based optical sensor calibration on the primary user. Optimal registration achieved with maintenance of moderately bright and diffuse lighting. The minimal hardware requirement for system development were presented as follows:

- $\quad$ Processor greater or equal to $1.8 \mathrm{GHz}$.

- 2 GB of RAM or 4 GB of RAM

- Minimal hard disk free installation space of 20-50 GB

- Installation of Windows and Visual Studio on a solid-state drive (SSD).

- $\quad$ Supported display resolution with a minimal of $720 \mathrm{p}$

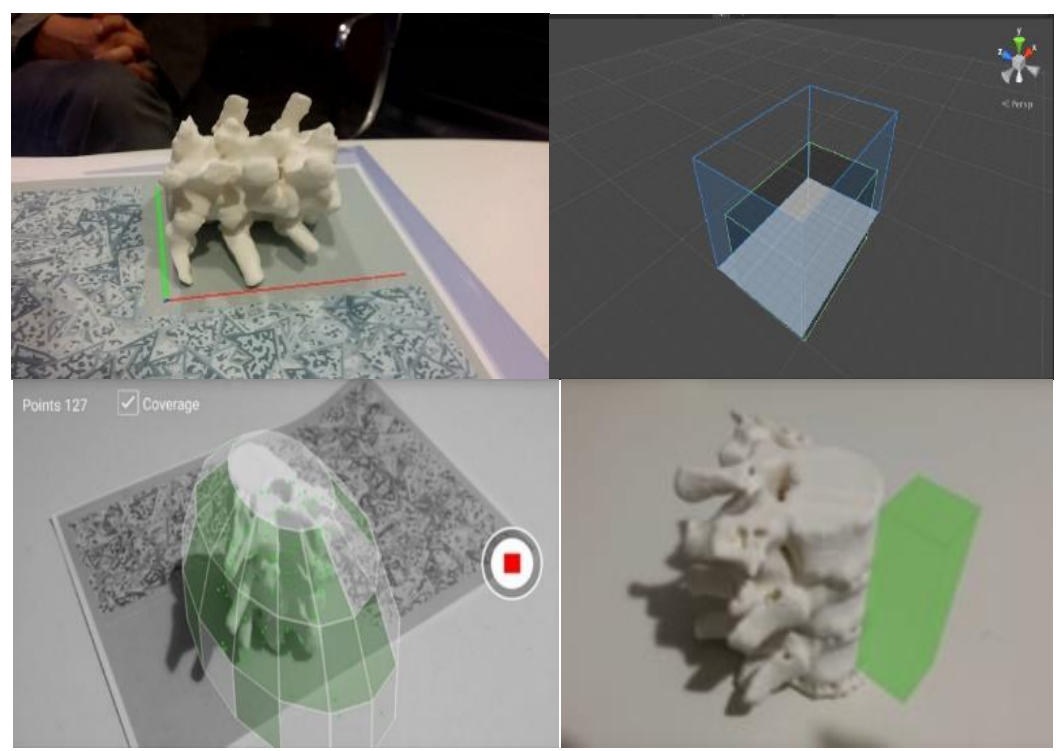

Fig. 1. Object target registration process.

\subsection{Target Registration}

Vuforia Object scanner produces the Object Data ( ${ }^{*}$.OD) file needed for reference identification. The Android Package Kit (APK) installer was accessible through Android Device Bridge (ADB) under the Tools section of Vuforia developer portal. The scanner pans around the intended object to overlay virtual pointers on its distinguishable features. Every completed registration ran through visual feedback and quality tracking evaluation before proceeding to Unity dataset conversion via target manager of the Vuforia developer Portal. Identifiable data result secures the placement of holograms.

The overall projection accuracy is dependent on the mobile optic quality in geometrical differentiation by the amount of the virtual pointers registered. Printed reference base shown in Fig. 1 served as the physical scale estimation for the intended object. Removal of unsupported surfaces such as featureless regions, pliable and articulating surfaces was done by culling. The feature distinguish items from other similar 
geometries. Local target origin is represented by $(0,0,0)$ at the lower left-hand corner of the grid region and corresponds to the local $(0,0,0)$ of the Object Target prefab instance's bounding box. The unit scale of the grid, Unity scene and estimated physical object scale were in millimeters.

\subsection{System Assembly}

Unity project development platform were used as the base program for system assembly. SDK sets and Object Data file were imported into Unity as assets. List of prefabs were filtered and assigned with parent prefabs that specialized in different conducts. Intended functionalities involves object target identification with additional user interactive functionalities. The first stage of system assembly involves AR and Hololens Camera placements. The stated cameras were used as indicators to command visual sensing upon the deployment of HoloLens. The point of projection directly correlates with arrangement of the model and image plane where visual references were available under scene panel. Furthermore, the best over layered fit of the projection relies on proper scaling of holographic projection done with minimal variation from the Object Target Scanner base.

\subsubsection{Project development platform}

The selection of Unity Editor as correlates with the offered features of level design and work building by the real-time rendering engine and Native graphics APIs. The platform provides multiple tools out in-on-editor of the box to support rapid editing and iteration in development cycles with Unity's instant play mode. Moreover, it has the most platform support than the other current creation engine and offers built-in solutions to optimize overall progress for professionals and amateur a like, the highlighted aids were:

- Revenue-generation solutions in forms of Ads \& IAP

- A complete set of live-operation analytics features for monitoring player activity.

- Continuous improvements in optimization of the development performance in real-time, without redeployment, or increase user Lifetime Value (LTV) with the power.

Any edition of the unity prior to the latest 2017.2.1f1 requires imports of Vuforia SDK to access target recognition features. Selection of the SDK packages are highly dependent on the unity engine used, as certain features were already imbedded into the system. Incompatibility triggers system crashes with detection of overlapping commands. For Unity engine version 2017.2.1f1, it integrates Vuforia engine to simplify the experiences in creation of cutting edge augmented reality applications by removing the import procedures. The implementation of Vuforia Augmented Reality Support is carried out during installation, in addition to the general platform support. Vuforia functions will be available in the Unity Game Object menu as well in the Build and Player Settings. However, to build a Vuforia App or use it in Play mode, XR settings for Vuforia requires activation. The player and quality components within project settings require alteration to better fit the deployment conditions.

\subsubsection{System development kit}

SDKs provides developers with integrated development environment (IDE) that represents the central programming interface. The IDE writes source code, incorporate debugger for error fixture, and grants access in creation of the program's graphical user interface (GUI) through a visual editor in program window. IDEs also include a compile that creates applications from source code files. The SDK package used were MixedRealityToolkit and Vuforia obtained from GitHub and Vuforia domain respectively. The object target feature was enabled once the prefab establishes link with HoloLens camera. The process involves activation of VR Support in XR Settings under configuration for stereo rendering and display support with addition tracking HoloLens tracking feaures.

MixedRealityToolkit implements gesture manipulation into project development as interactive command 
inputs. There were several different forms of prefabs that focus on manipulation and navigation instructive controls. The cursor prefab grants user hologram interactive access under familiarized cursor operatives. It may be visually distinctive to reflect the current system state. The cursor navigates with head motion and output commands based on the received verbal or gesture feedbacks. The attached C\# scripts detailed the intended target outcome. Cursor with feedback applications, universal interactive button, raw interaction input, Gaze manage, and stabiliser were some of the command script attached. The axis controller script offered the projected model a directional guide along the axis. In addition, spatial mapping command was implemented to the project to better distinguish target by disregarding surrounding objects.

\subsection{HoloLens Deployment}

The system completed assembly process once all collective scenes went through Unity build settings. Selection of Build for Universal Windows Platform will generate a native project that is executable from Visual Studio. Capabilities enabled for publish player settings were internet client, pictures library, videos library, web cam, microphone, human interface device, objects 3D and spatial perception to notion HoloLens the system's list of deployment requirement.

The finalized program system was deployed via Visual Studio Community 2017. Visual studios contains developer tools and services targeted for app building purposes with adaptation of any platform. The list of available tools involved debugging, code navigation, IntelliSense, refactoring, and code hints used to maximize development efficiency. Selection of either one will be sufficient. The Universal Window Platform development workload and the Game Development with Unity workload is the 2 essential options required for system development. Absence of either one will trigger the error CS0006 - absence of metadata file which result in failure in deployment.

\subsection{Accuracy Verification}

An ideal HoloLens application shows a consistent production of minimal $60 \mathrm{fps}$ for a singular frame latency to optimize general user comfort. The HoloLens display pipeline makes minor alterations on the presented application frames to upscale the frame rate to match the display's native frame rate. The accuracy verification process were done on the recording of the application in function, as in Fig. 2. Target object placed on flat surface to create stationary coordinate reference. The overall verification process are listed as follows:

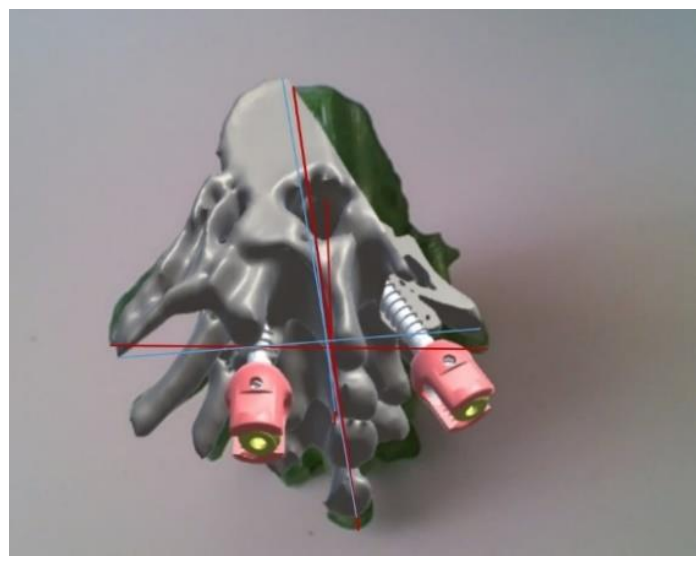

Fig. 2. Accuracy verification.

1. 80 frames generated under a second collected with coordinate system imposed for both hologram projection (blue) and physical target (red). 
2. For distance error, record and substitute the projection coordination $\left(P_{i x}, P_{i y}, P_{i z}\right)$ of each frame with the relative target coordination $\left(P^{*}{ }_{i x}, P^{*}{ }_{i y}, P^{*}{ }_{i z}\right)$ into the provided equation below.

$$
P_{i}=\sqrt{\left(P_{i x}-P^{*}{ }_{i x}\right)^{2}+\left(P_{i y}-P^{*}{ }_{i y}\right)^{2}+\left(P_{i z}-P^{*}{ }_{i z}\right)^{2}}, \quad(i=0,1,2 \ldots .80)
$$

3. For angular error, record and substitute the projection rotation $\left(A_{i x}, A_{i y}, A_{i z}\right)$ of each frame with the relative target rotation $\left(A^{*}{ }_{i x}, A^{*}{ }_{i y}, A^{*}{ }_{i z}\right)$ into the provided equation below.

$$
\begin{aligned}
& A_{i}=\cos ^{-1}\left[A_{i x} \times A^{*}{ }_{i x}+A_{i y} \times A^{*}{ }_{i y}+A_{i z} \times A^{*}{ }_{i z} / \sqrt{A_{i x}{ }^{2}+{A_{i y}}^{2}+{A_{i z}}^{2}} * \sqrt{{A^{*}{ }_{i x}{ }^{2}+{A^{*}}_{i y}{ }^{2}+{A^{*}}^{*}{ }^{2}}^{2}}\right] \\
& (i=0,1, \ldots .80)
\end{aligned}
$$

\section{Result}

The final application succeeded in visual target identification and data registration. The hologram projection clung to a particular geometry feature of the chosen object. Hence, any movement carries insignificant effects upon overall deployment relay as the projection were set to automatically calibrate and mimic the physical object placement, as in Fig. 3.

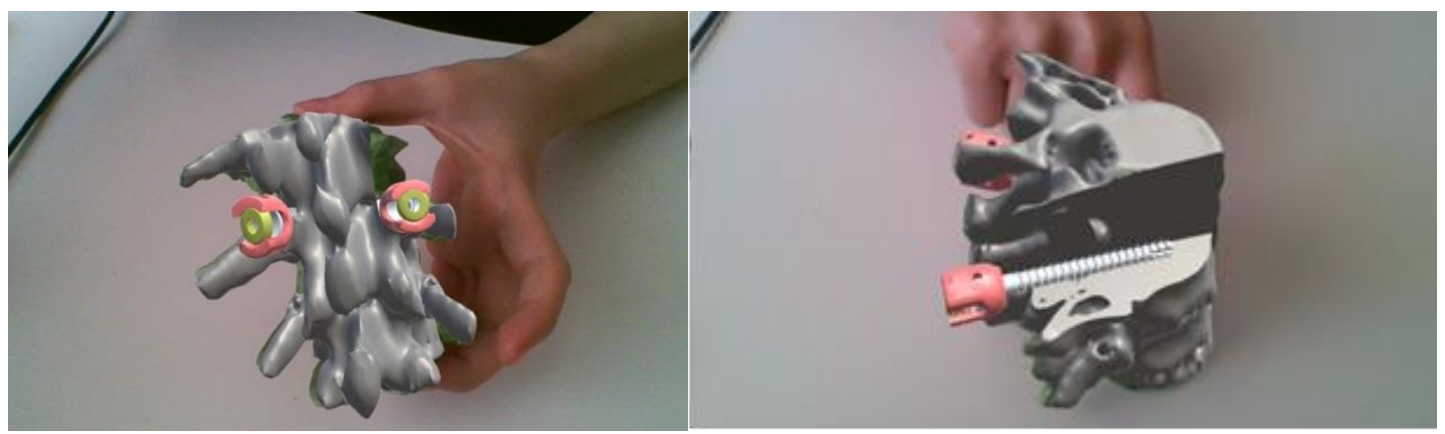

Fig. 3. Spine projection overlay.

Further observation also noted that once the previous anchored feature were hid out of sight, the system will drops to $30 \mathrm{fps}$ before relocates to another geometry feature as the new target anchor. A variety of UI application were included under focus for pre-surgical planning and to minimize reliance of other supporting equipment. The lumber screws shown in Fig. 3 were not a part of the 3D-printed spine model

\begin{tabular}{|c|c|c|c|c|c|c|}
\hline \multirow[t]{2}{*}{ Frame } & \multicolumn{3}{|c|}{ Maximum Distance Error (mm) } & \multicolumn{3}{|c|}{ Maximum Angular Error $\left({ }^{\circ}\right)$} \\
\hline & $\mathrm{x}$ & $\mathrm{y}$ & $\mathrm{z}$ & $\mathrm{x}$ & $\mathrm{y}$ & $\mathrm{z}$ \\
\hline $1-20$ & 0.00 & 0.00 & 0.00 & 5.00 & 3.00 & 5.00 \\
\hline $21-40$ & 1.00 & 2.00 & 0.00 & 2.00 & 4.00 & 2.00 \\
\hline $41-60$ & 0.00 & 1.00 & 0.00 & 4.00 & 4.00 & 4.00 \\
\hline $61-80$ & 2.00 & 1.00 & 0.00 & 4.00 & 5.00 & 5.00 \\
\hline Mean & 0.30 & 0.36 & 0.03 & 0.95 & 0.81 & 1.28 \\
\hline
\end{tabular}
and could be manipulated at will through hand gestures and audio commands.

Table 1. Accuracy Estimation

The accuracy estimation for 80 fps operation shown maximum mean distance error of $0.36 \mathrm{~mm} \pm$ $0.10 \mathrm{~mm}$ while with maximum angular error of $1.28^{\circ} \pm 0.50^{\circ}$ among 3 axis. Table 1 indicates the application capability in restraining generated error under $0.4 \mathrm{~mm}$ and $1.3^{\circ}$ each second without any spike upon overall error distribution. The results solidifies the adaptability of the HoloLens application upon 
clinical pursuits.

\section{Discussion}

Microsoft HoloLens provided an interesting perspective to AR fusion surgical application, and allowance of SDKs expanded the system capability. Object target referencing defers from others by the sole reliance of target geometrical features. The registration process utilizes fundamental core building block of spatial mapping, coordinate system and spatial anchor. Gaze - primary form of target input - collaborates with system cursor to ensure accurate instructive commands. Default gaze vectors prioritize target sizes within 1 to 1.5 degrees. Calibration of UI elements were advised to be around eye level to leverage the attention-chaining behaviour while mapping the surrounding components. The incorporated gaze stabilization function removes the behavioural induced visual jitters or wobbles.

Holographic frame were used to highlight and restrain general user focus on a particular application. Seated-scale experiences could be built through the stationary frame of reference. Centre of origin in Unity program directly correlates to the user's head position and orientation. Objects were then placed at a specific coordination in reference of the stationary frame. An app will typically create one stationary frame of reference on start-up and use its coordinate system throughout the app's lifetime. Spatial mapping utilizes the frame to constrain hologram placements from "point in space" (3D) to "point on surface" (2D) in simulation of user interactive familiarity. Optimal surface tracing results highlighted the importance of traditional lighting upon mesh properties. Precautions and regulations were implemented to eliminate result compromising factors. Head mounted displays in general were vulnerable to light as image detail processing requires an environment with ambient lighting.

The effect of activating the extended tracking box to initialize target tracking is that the target tracker will identify the target object and analyze the position and rotation of the target for a robust pose estimation for HoloLens use. HoloLens will initiate with Vuforia tracker deactivated after Vuforia engine transform the dataset content into the HoloLens spatial mapping coordinate space. However, flickering of projection model was shown and the scale of the projection was not exact. Similar to the later discussed issue encountered with implementation of UI buttons, the cause of the flickering might be caused by the change between highlighted and pressed appearance of the object. The simple solution for this issue is to deploy the hologram in an even lighted room. Aside from repetitive testing to obtain the proper scale, Unity editor edition 2017.2 could be used as it provides real-time calibration access to the developers. However, it would indicate that the whole program would have to be reconstructed due to incompatibility of the scripts used.

Sensor tuning would be a step further from calibration that requires longer process time. Response of hand gesture will be short and precise for single-click motion acted upon cursor hovered object. The "Time To Press On Tap" field of the HoloLens Input Module component affects the response time of user interactive components. Thus, providing users with better feedback response about leaving UI object on hold. As navigation gestures are crucial to the program development, the "Normalized Navigation To Screen Offset Scalar" field of "HoloLensInputModule" dictates the pixels number of a cursor in motion for a navigation gesture where the device reported a positive or negative 1value. If a slider object is ever involved, slight flickering would be apparent upon the deployment of the project. This might be caused by the change between Highlighted and Pressed appearance of the object. However, the navigation gesture would still be functional disregarding the apparent"

A known issue of HMD was the discomfort induced from wearing the device for an extended period of time. User symptoms ranged from muscle sore to lightheadedness. Adapting any light weight HMDs such as the Magic Leap or Google glass is advised as the final program is adaptable to either devices. For those who struggle to secure the device, detachable headset accessories would have provided additional support. 


\section{Conclusion}

We experimented with applying HoloLens to assist in spinal fusion surgery with a focus object target registration. We found that navigation and manipulation through interactive command gestures were concise and fluid, which indicates the versatility of the Microsoft HoloLens device. The results indicate great promise in probable adaptation under infra-operative clinical field.

\section{References}

[1] Ewers, R., Schicho, K., Undt, G., et al. (2005). Basic research and 12 years of clinical experience in computer-assisted navigation technology: A review. Int J Oral Maxillofac Surg.

[2] Jeffs, G. J., Lee, G. Y. F., \& Wong, G. T. H. (2003). Functioning paraganglioma of the thoracic spine: Case report. Neurosurgery, 53(4), 992-995.

[3] Lee, G. Y. F., Massicotte, E. M., \& Raja, R. Y. (2007). Clinical accuracy of cervicothoracic pedicle screw placement: A comparison of the "open" lamino-foraminotomy and computer-assisted techniques. Journal of Spinal Disorders \& Techniques, 20(1), 25-32.

[4] Paradiso, G., Lee, G. Y. F., Sarjeant, R., et al. (2006). Multimodality intraoperative neurophysiologic monitoring findings during surgery for adult tethered cord syndrome: Analysis of a series of 44 patients with long-term follow-up. Spine (Phila Pa 1976). Spine, 31(18), 2095-2102.

[5] Ezoji, M., \& Faez, K. (2011). Use of matrix polar decomposition for illumination-tolerant face recognition in discrete cosine transform domain. IET Image Processing(2011), 5(1), 25.

[6] Foley, K. T., Simon, D. A., \& Rampersaud, Y. R. (2001). Virtual fluoroscopy: Computer-assisted fluoroscopic navigation. Spine, 26(4), 347-351.

[7] Mischkowski, R. A., Zinser, M. J., Kübler, A. C., et al. (2006). Application of an augmented reality tool for maxillary positioning in orthognathic surgery - A feasibility study. Journal of Cranio-Maxillofacial Surgery, 34(8), 478-483.

[8] Vassallo, R., Rankin, A., Chen, E. C. S., \& Peters, T. M. (2017). Hologram stability evaluation for Microsoft (R) HoloLens TM. Medical Imaging.

[9] Alaraj, A., Charbel, F. T., Birk, D., et al. (2013). Role of cranial and spinal virtual and augmented reality simulation using immersive touch modules in neurosurgical training. Neurosurgery, 72(1), 115-123.

[10] Lindeque, B. G. P., Ponce, B. A., Menendez, M. E., et al. (2014). Emerging technology in surgical education: Combining real-time augmented reality and wearable computing devices. Orthopedics, 37(11), 751-757.

[11] Wang, M. L., Vemuri, A., Ho, Y., et al. (2013). Hybrid optical and electromagnetic method for medical instrument tracking application. Proceedings of the International Symposium on Consumer Electronics, ISCE.

[12] Aschke, M., Wirtz, C. R., Raczkowsky, J., et al. (2003). Augmented reality in operating microscopes for neurosurgical interventions. Proceedings of the International IEEE/EMBS Conference on Neural Engineering, NER.

[13] Inoue, D., Cho, B., Mori, M., et al. (2013). Preliminary study on the clinical application of augmented reality neuronavigation. J Neurol Surg A Cent Eur Neurosurg 2013, 74(02), 071-076.

[14] Chang, Y. Y., Yan, S. L., Lin, P. Z., et al. (2015). A mobile medical QR-code authentication system and its automatic FICE image evaluation application. Journal of Applied Research and Technology, 13(2), 220-229.

[15] Citardi, M. J., Agbetoba, A., Bigcas, J.-L., \& Luong, A. (2016). Augmented reality for endoscopic sinus surgery with surgical navigation: A cadaver study. Int Forum Allergy Rhinol, 6(5), 523-528.

[16] Chiorboli, G., \& Vecchi, G. P. (1993). Comments on "Design of Fiducials for Accurate Registration Using 
Machine Vision." IEEE Trans. Pattern Anal. Mach. Intell.

[17] Hanna, M. G., Ahmed, I., Nine, J., et al. (2018). Augmented reality technology using microsoft holoLens in anatomic pathology. Arch Pathol Lab Med, 142(12).

[18] Eliashar, R., Sichel, J.-Y., Gross, M., et al. (2003). Image guided navigation system-A new technology for complex endoscopic endonasal surgery. Postgrad Med J.

[19] Chen, H., Lee, A. S., Swift, M. ,\& Tang, J. C. (2015). 3D collaboration method over holoLens ${ }^{\mathrm{TM}}$ and skype ${ }^{\mathrm{TM}}$ end points. Proceedings of the 3rd International Workshop on Immersive Media Experiences ImmersiveME'15.

[20] Ghosh, A., \& Nirala, A. K. (2014). One to one imagery using single hololens configuration. Opt Rev, 21(6), 765-768.

[21] Craig, A. B. (2013). Understanding augmented reality: Concepts and applications. Underst Augment Real.

[22] Kersten-Oertel, M., Jannin, P., \& Collins, D. L. (2013). The state of the art of visualization in mixed reality image guided surgery. Comput Med Imaging Graph, 37(2), 98-112.

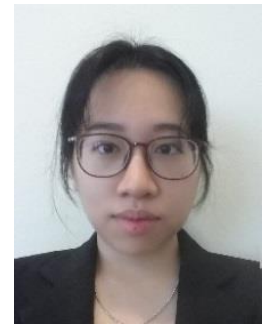

Pei Wen Voon recieved her BSc in mechanical engineering from Curtin University, Perth, Australia, in 2017. She is preparing for her Ph.D pursuit in mechanical engineering at Curtin University, Perth, Australia for head mounted display surgical adaptation. Her technical interest include AR target registration refinement and image processing.

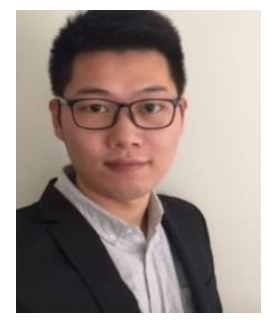

Ke Wang received his Ph.D. in mechanical engineering from Curtin University, Perth, Australia, in 2018. His research interests lay in building practical robotic systems using sophisticated sensors and sensing algorithms. He is also interested in designing sensing systems using bionic or biomimicry methodology.

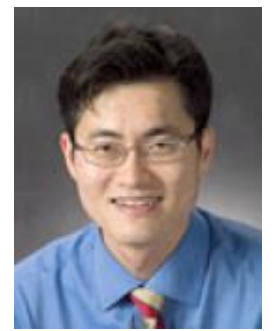

Gabriel Lee was born in Kuching, Sarawak in 1972. He received his MBBS (Hons) and MS degrees from the University of Adelaide, South Australia. He completed his specialist training in Neurosurgery after relocating to Western Australia and became a fellow of the Royal Australasian College of Surgeons in 2003. He has dual post-graduate clinical fellowships in Spine Surgery (Toronto Western Hospital, Canada) and Epilepsy Surgery (Yale-New Haven Hospital, Connecticut, USA).

He has been working as a consultant neurosurgeon at SJOG Subiaco Hospital and Sir Charles Gairdner Hospital since 2006. He is a clinical professor affiliated with both University of Western Australia and Curtin University. He has been extensively involved in undergraduate medical student and post-graduate neurosurgery teaching. His research interests include neuro-oncology, spinal disorders and development of medical technologies including spinal instrumentation and neurosurgical navigation. 


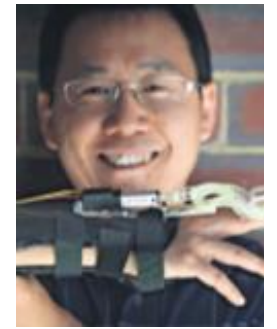

Lei Cui is a roboticist who designs and develops novel robots and sensing technologies for various applications. His research has been motivated by the belief that rigorous mathematical results will enable engineering advancements while the practical aspects of implementation will drive theoretical progress. The goal of his work is to create sophisticated and autonomous robotic systems. He received his PhD degree from King's College London (UK), worked at Carnegie Mellon University (US), and is currently senior lecturer at Curtin University (AU). 